\title{
Use of Convolutional Neural Network for Fully Automated Segmentation of Hard Exudates in Retinal Images
}

\author{
By: Aidan Lochbihler \\ $2^{\text {nd }}$ Year Biomedical \& Electrical Engineering, \\ Carleton University
}

March $7^{\text {th }}, 2019$

Inquiry@ Queens 


\section{Introduction}

- Purpose: describe a fully automated deep learning-based method to segment hard exudate from retinal images of patients with Diabetic Retinopathy

- Convolutional Neural Network consists of an input and an output layer, as well as hidden layers in between

- Implementation of a U-Net-based convolutional neural network (CNN) based model

- The real world applications of cutting edge machine learning techniques in the medical field 


\section{Diabetic Retinopathy (DR)}

- One of the leading causes of blindness and visual impairments worldwide, especially in developing countries

- DR affects $80 \%$ of patients with diabetes and is the leading cause of blindness around the world [1]

- If Diagnosed early can be treated and prevented

- Hard exudates (HE): one type of lesion that appears as sparse yellowwhite region on retinal images with the varying size

- HE was chosen since it is often the more prominent lesion type in DR patient retinas 


\section{Method}

(C) A. Lochbihler 2019 Carleton 


\section{Training}
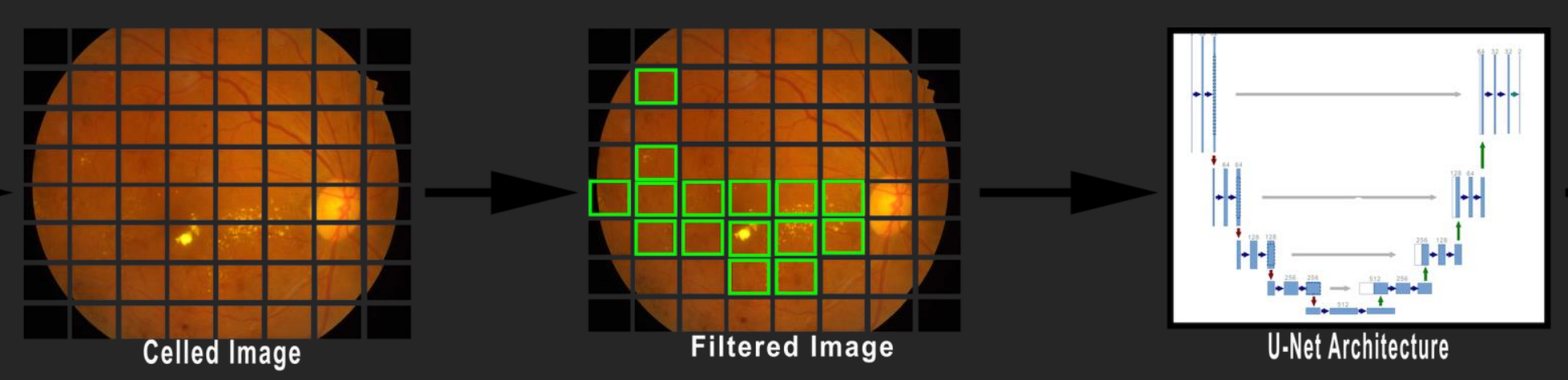
Convolutional
U-Net
Hard Exudate Model




\section{Input Image}




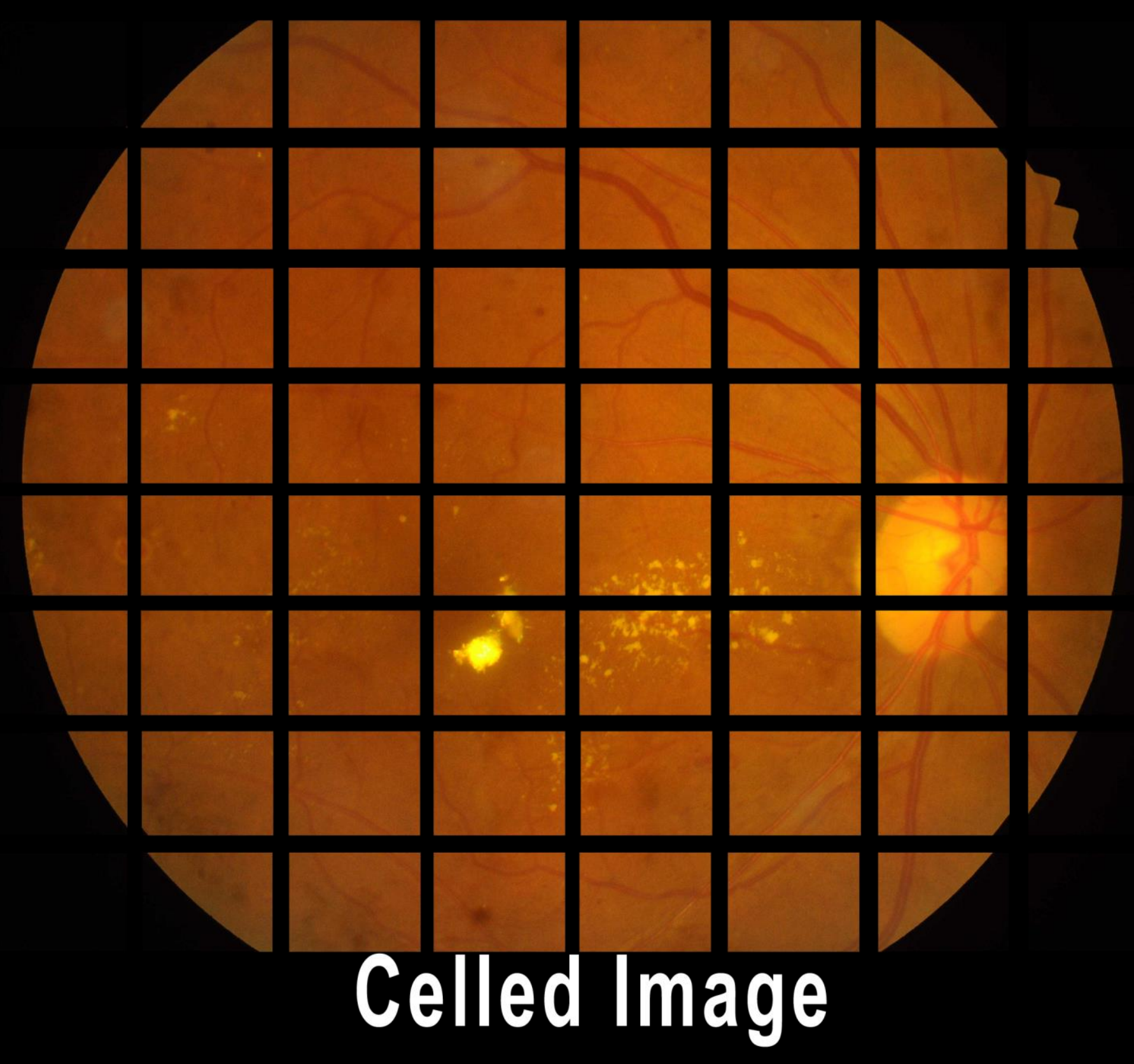




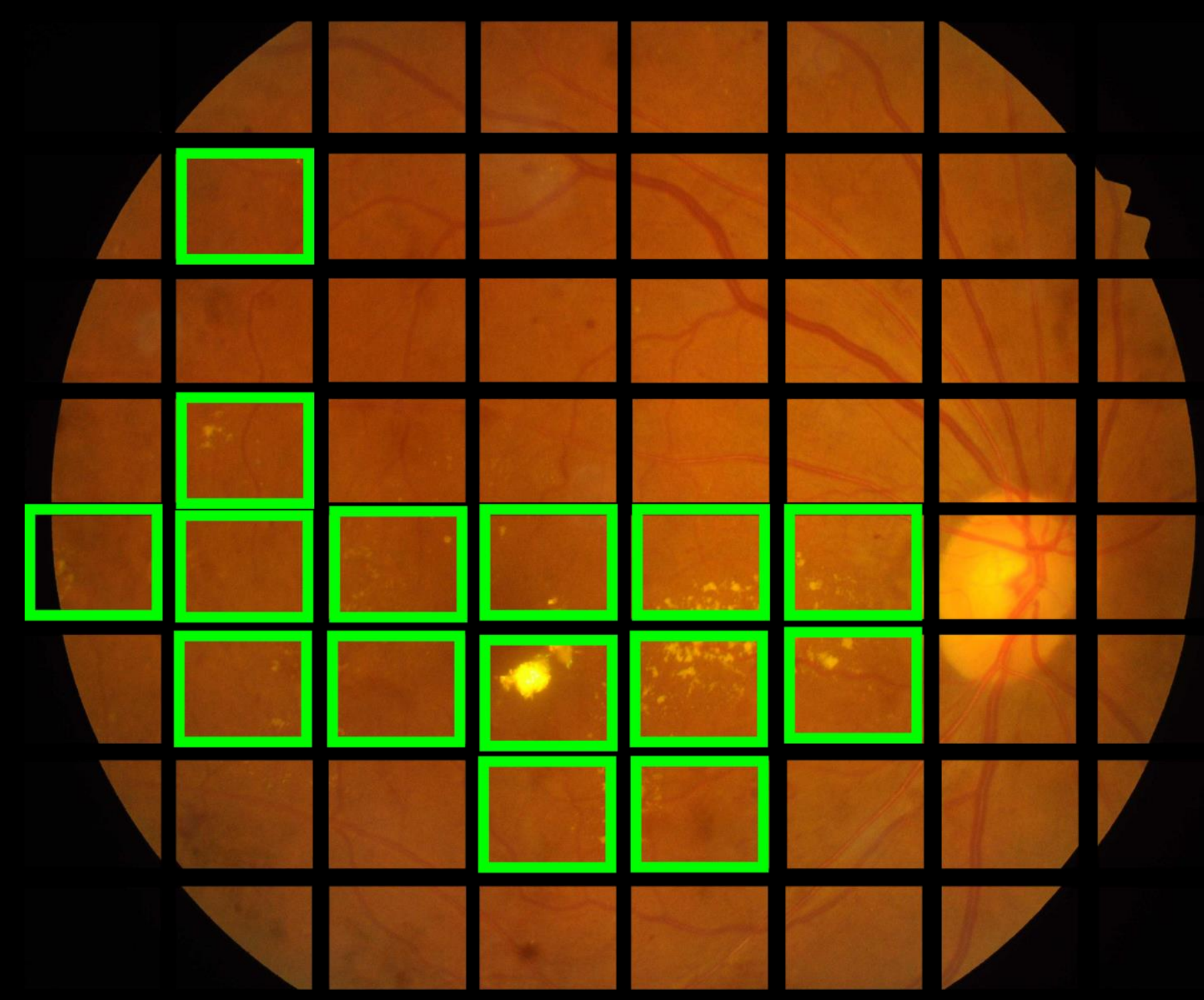

Filtered Image 


\section{U-Net Architecture}

\section{What is the U-Net?}

- Passes images through shrinking and expanding layers in order to learn patterns and features

- A convolutional network architecture for fast and precise segmentation of images

\section{Specifics of U-Net Model}

- Filter Size of 32

- Dice Coefficient $=\frac{2 *\left|X_{1} \cap X_{2}\right|}{\left|X_{1}\right|+\left|X_{2}\right|}$

- ELU

- Data Augmentation (Rotating, Shifting)

- Batch Normalization (Reduces overfitting) [2]

- Fully Convolutional

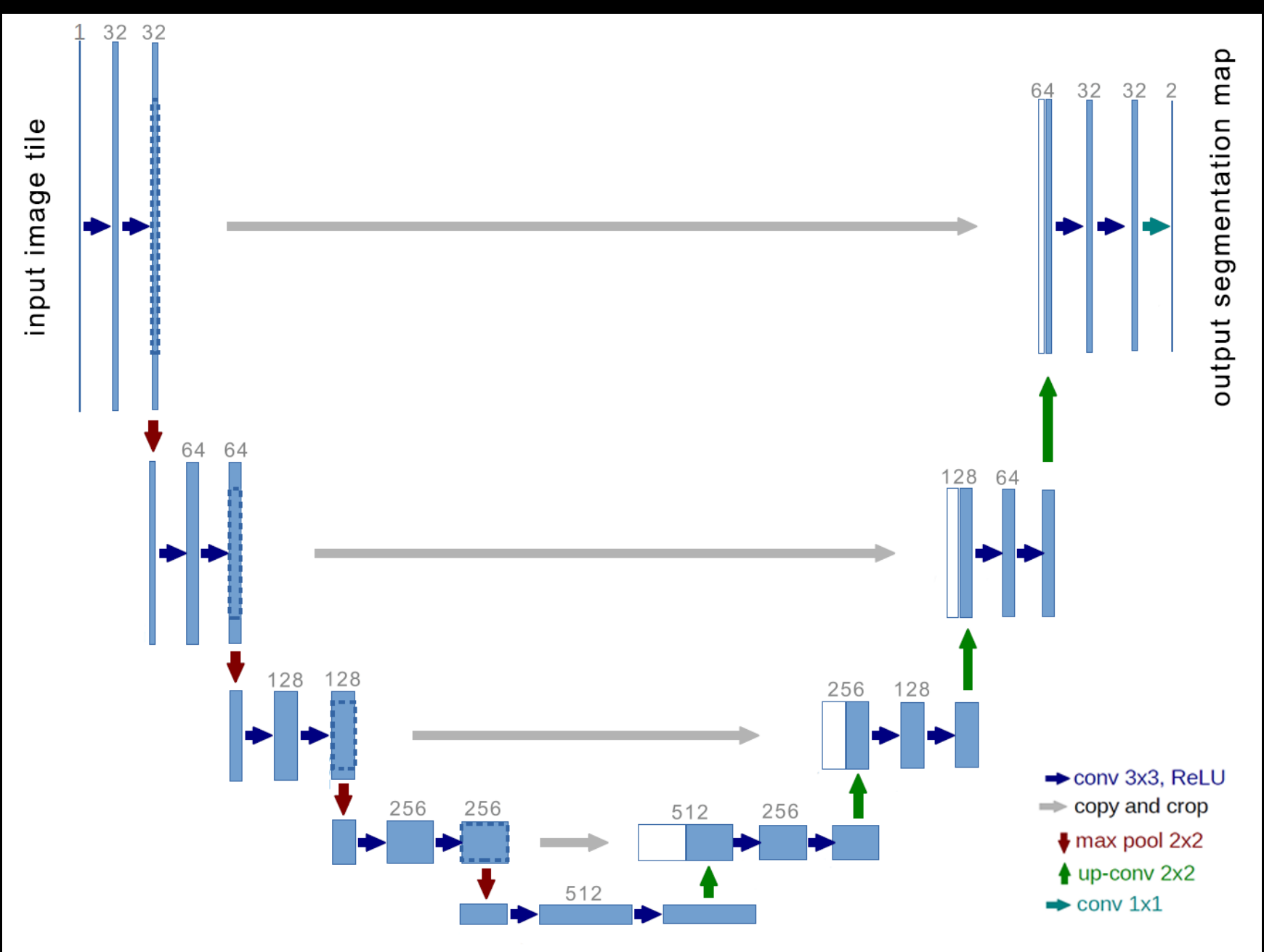

[2] U-Net Architecture 
Convolutional

U-Net

Hard Exudate

Model 


\section{Testing}

Convolutional

U-Net

Hard Exudate

Model

Celled Image

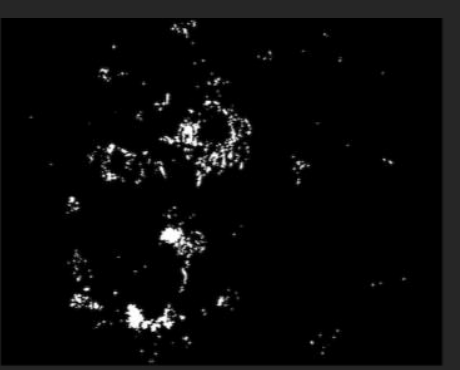

Output Binary Mask

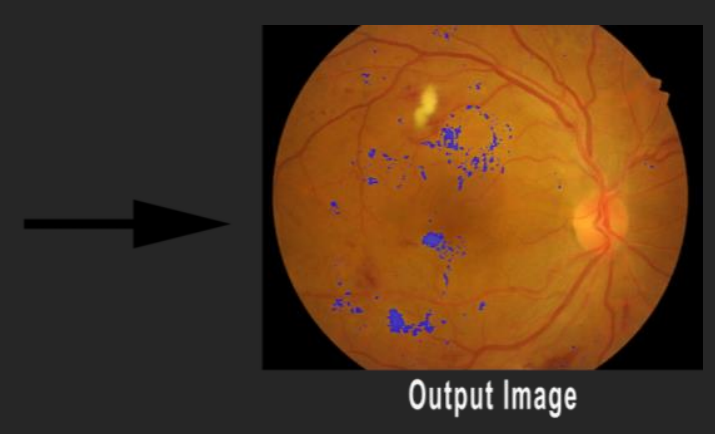

(C) A. Lochbihler 2019 


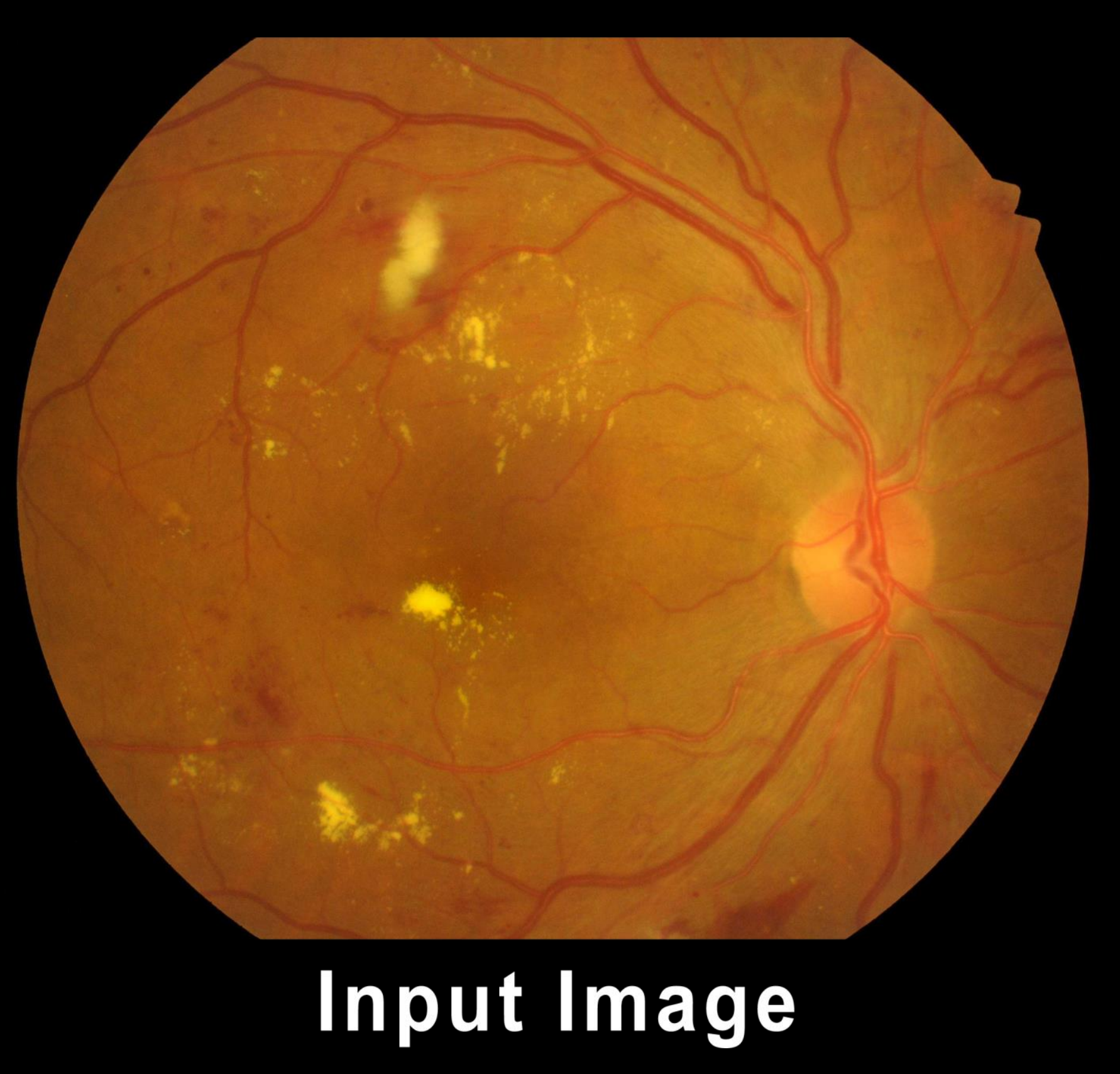




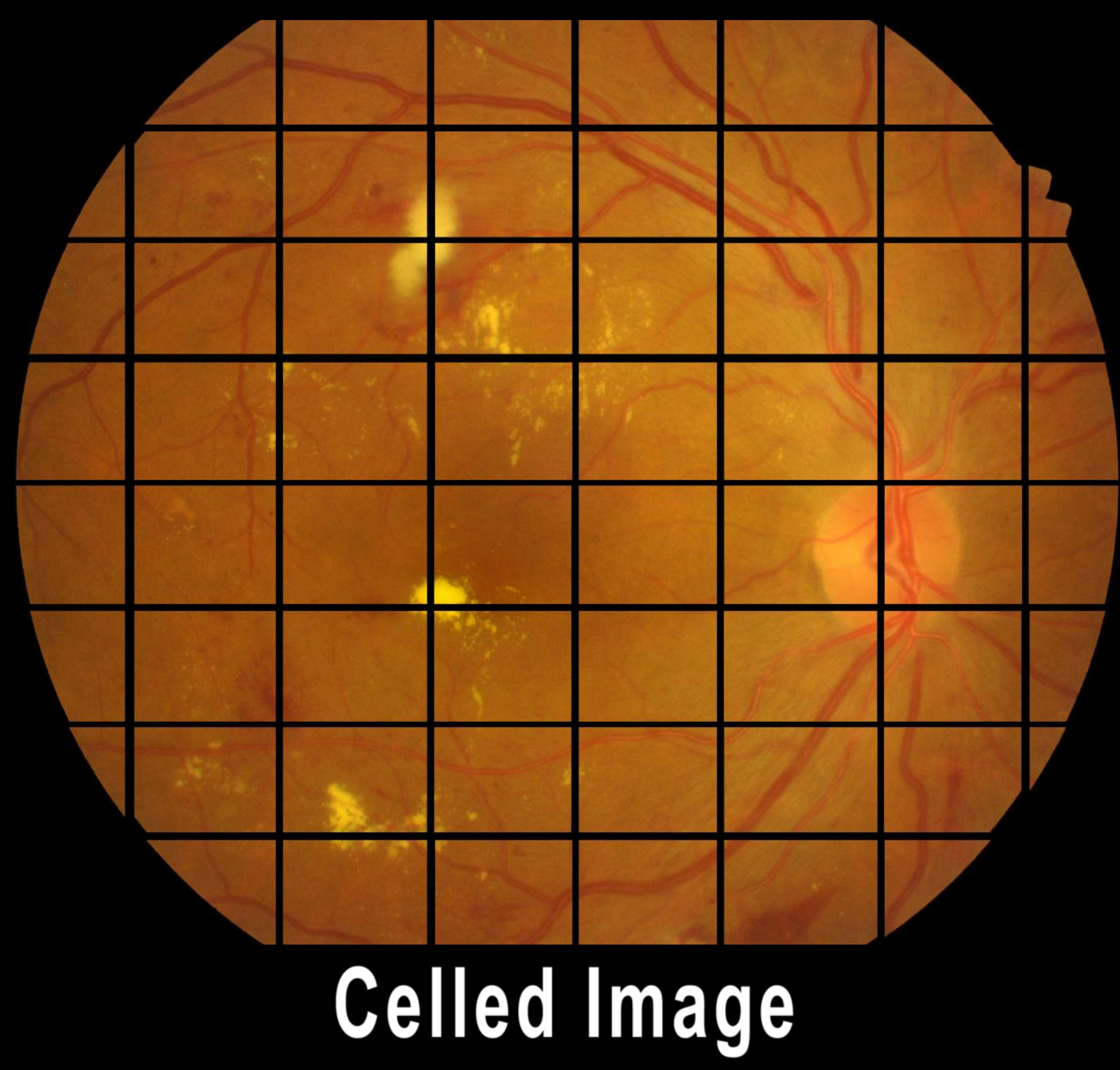


Convolutional

U-Net

Hard Exudate

Model 


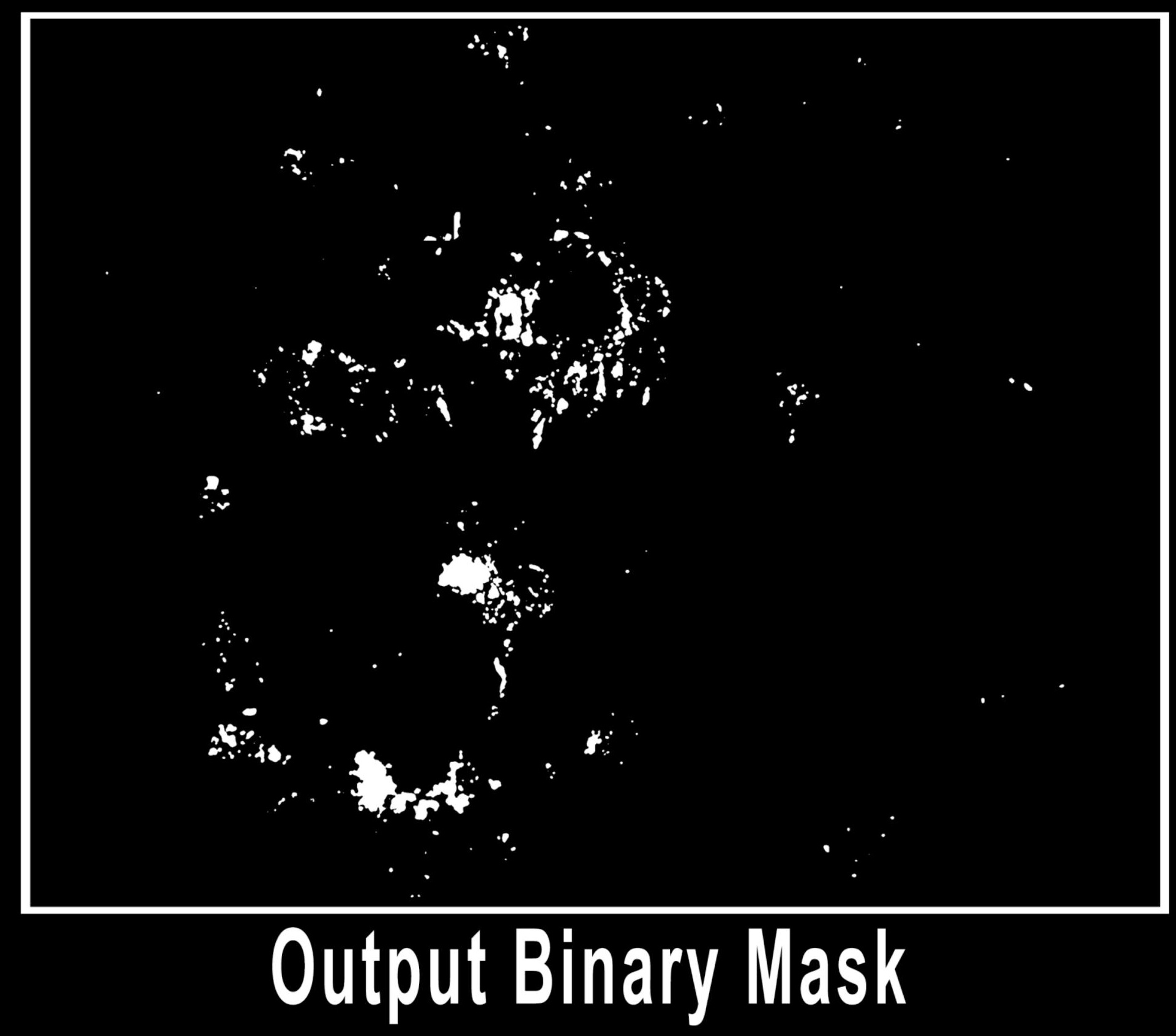




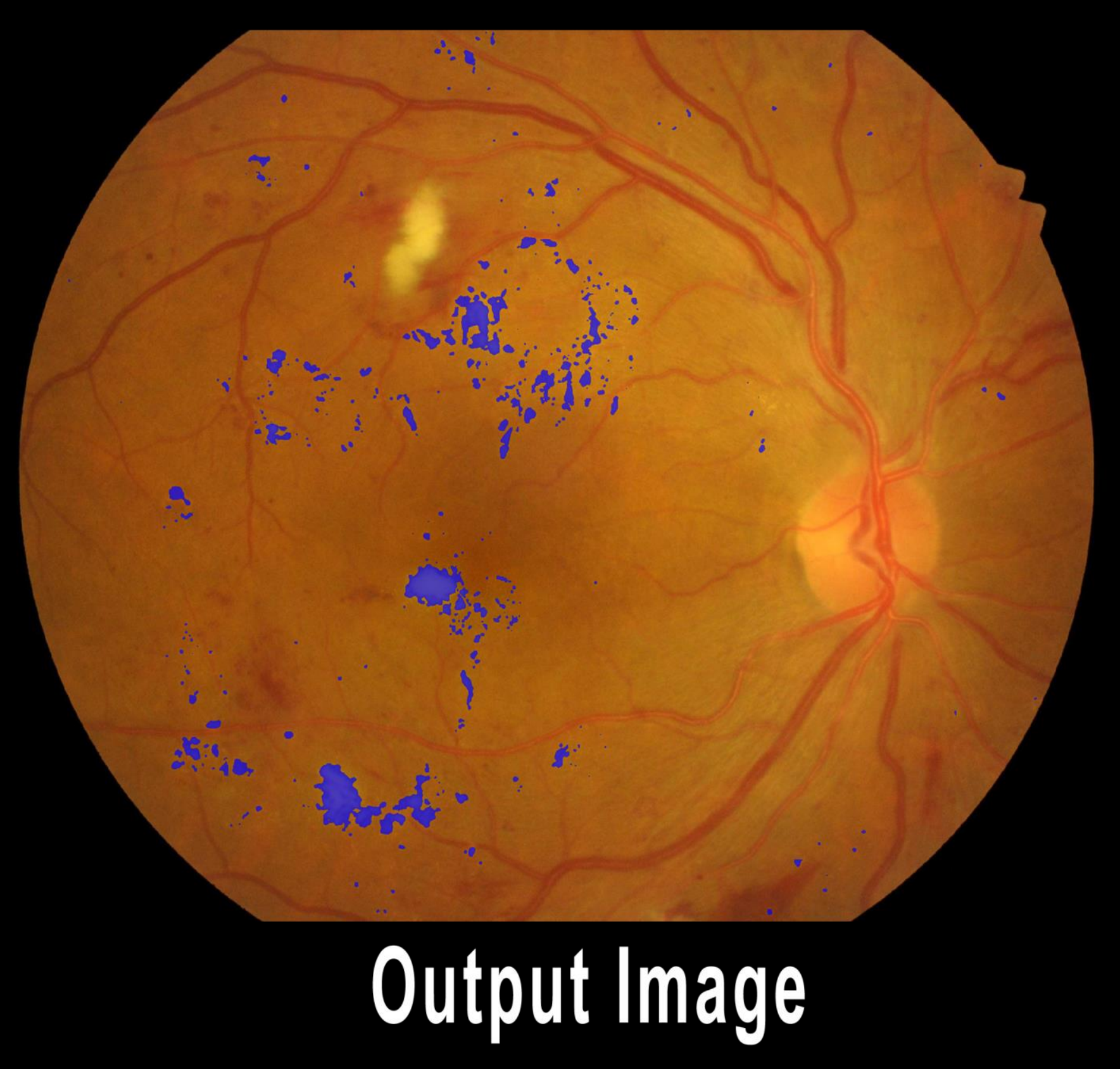




\section{Results}

(C) A. Lochbihler 2019

Carleton 


\section{Metrics Used}

- True Positive/Negative, False Positive/ Negative

- TP is a correct delineation of a lesion

- TN is a correct delineation of a non-lesion pixel

- FP is an incorrect delineation of a lesion pixel (is supposed to be a non lesion)

- FN is an incorrect delineation of a non-lesion pixel (is supposed to be a lesion)

- Dice Coefficient $\quad D I C E=\frac{2 *\left|X_{1} \cap X_{2}\right|}{\left|X_{1}\right|+\left|X_{2}\right|} \quad \begin{aligned} & \mathrm{x}_{1} \text { - the auto generated matrix } \\ & \mathrm{x}_{2} \text { - the ground truth matrix }\end{aligned}$

- Precision $\quad P P V=\frac{T P}{T P+F P}$

- Accuracy $\quad A C C=\frac{T P+T N}{P+N}$

- Specificity (True Negative Rate) $\quad T N R=\frac{T N}{T N+F P}$

- Sensitivity (True Positive Rate) $\quad T P R=\frac{T P}{T P+F N}$ 


\section{Statistical Evaluation}

- 26 Test Images

- I.e. 26 retinal images that the model was not trained on and has never seen before

\section{Metric 1: Comparing Auto Generated Mask to Ground Truth Mask}

- Dice $=67.23 \pm 13.60 \%$

- Specificity $=99.74 \pm 0.35 \%$

- Precision $=\mathbf{7 5 . 8 7} \pm \mathbf{1 8 . 1 4 \%}$

\section{Metric 2: Detection Rate}

- Output Masks were summed and if they were greater than a unit sum of 8500 they were designated as unhealthy and at risk

- Sensitivity (True Positive Rate) $=\mathbf{9 6 . 1 5 \%}$

- Meaning only 1 of the 26 images was falsely marked as healthy

- Overall Accuracy $\mathbf{8} \mathbf{8 8 . 4 6 \%}$

- This is the most important metric since it evaluates the models performance as it would be desired in the real world 

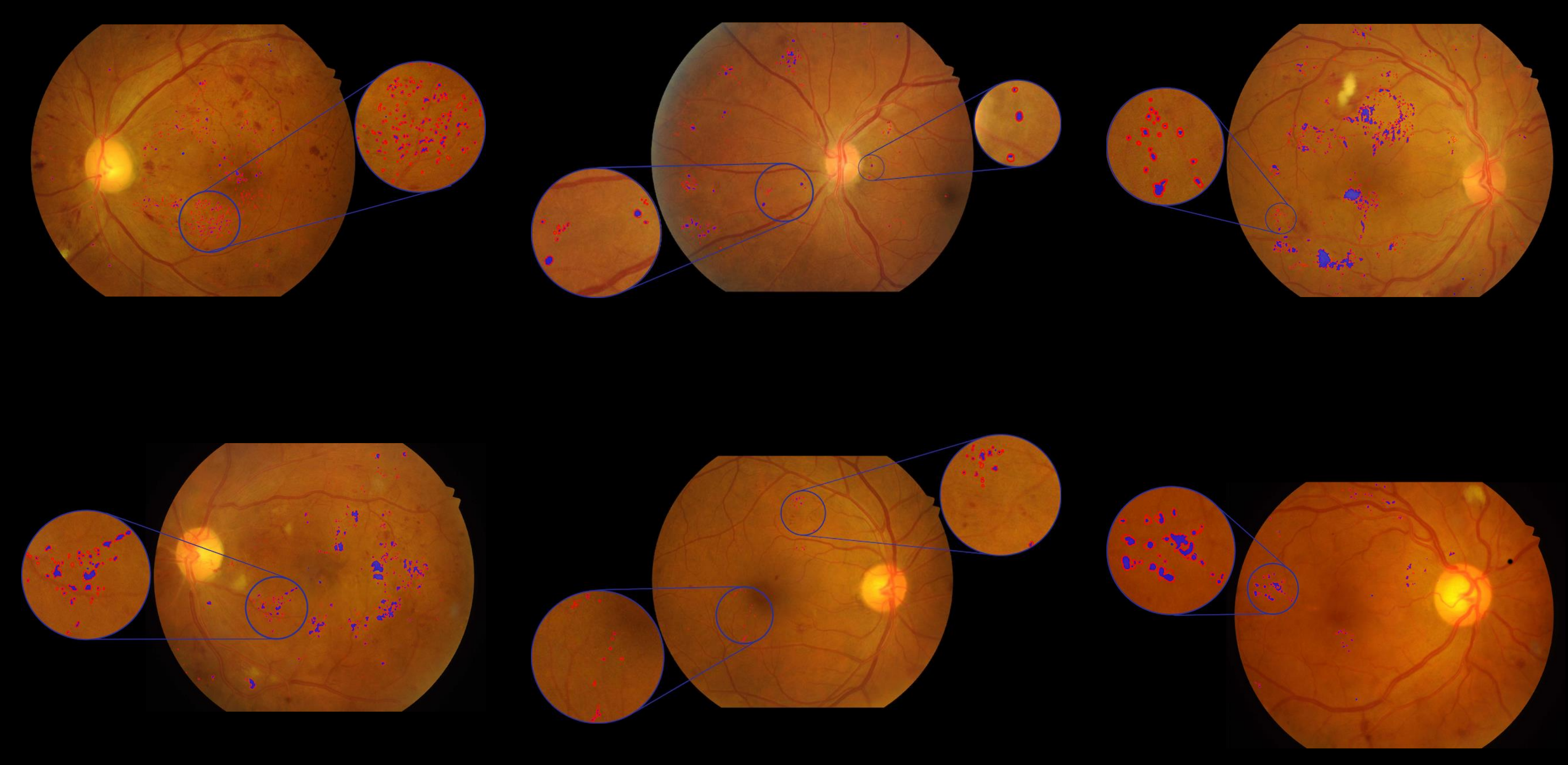


\section{Overall Detection Ability}

Blue: Automatically Generated Mask Red: Ground Truth Mask
更

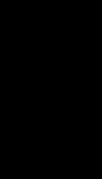




\section{Detecting Fine Details}

Blue: Automatically Generated Mask

Red: Ground Truth Mask

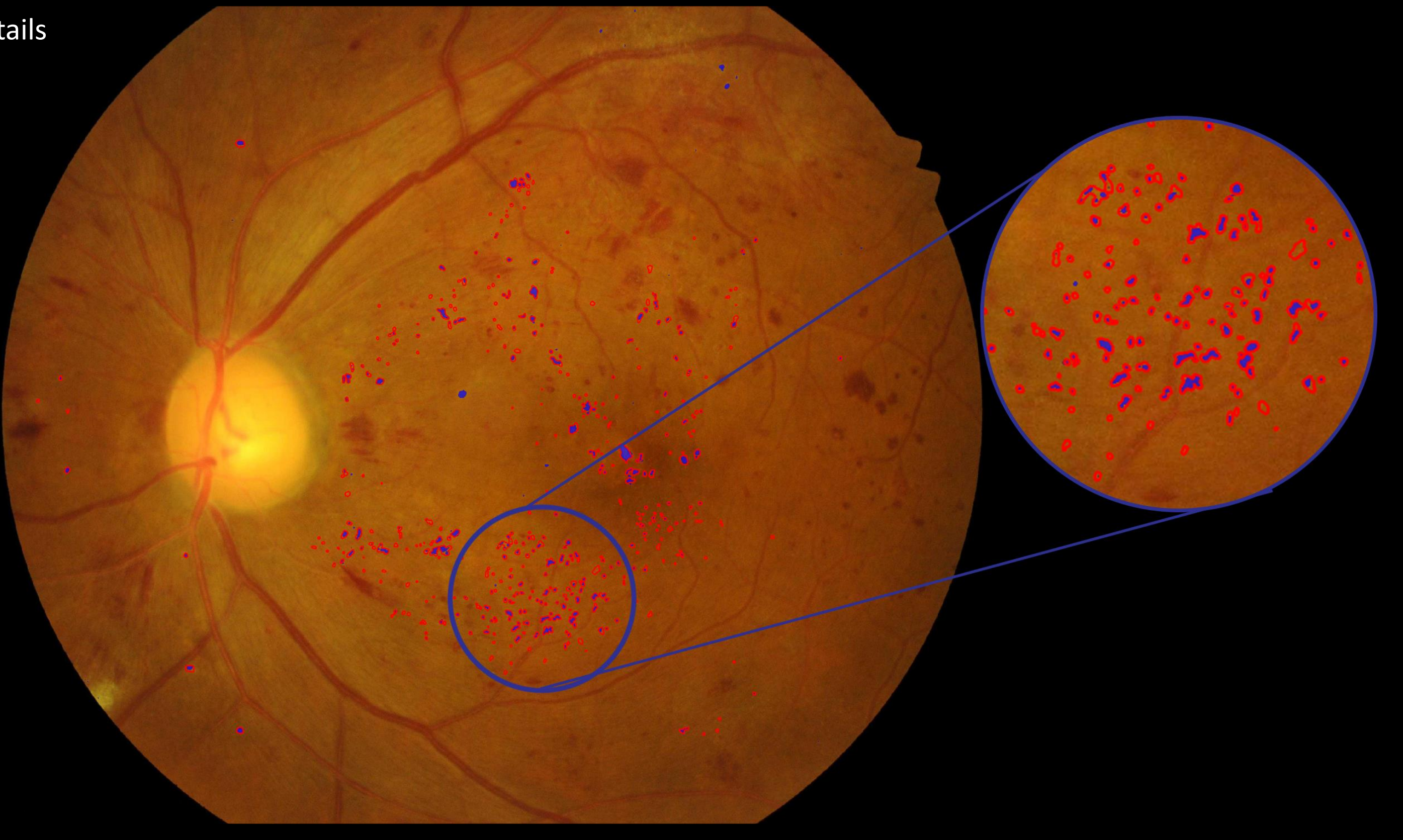




\section{Future Improvements}

- Future improvements of the model

- Creating a model that will be able to diagnose and classify all types of lesions that diabetic retinopathy can cause (5 types)

- Summing of the matrix can determine severity of DR

- Implementing the model into an eye scanner to give you immediate results 


\section{Conclusions}

- Key technology for revolutionizing the medical industry

- High potential for growth and improvement

- Hardware improvements benefit it greatly (decreases processing time)

- Popularization of the technology will only further quicken its advancement

- Will benefit the economy, our lives and many more aspects of the world

- Allowing for more patient doctor face to face time 


\section{Acknowledgments}

Supervisors: Fatemeh Zabihollahy ${ }^{1}$, Eranga Ukwatta ${ }^{1,2}$

Data: Public Indian diabetic retinopathy image dataset (IDRiD)

Sponsor: I-CUREUS Discovery Centre Carleton University (Aleksandra Minic) 


\section{References}

[1] P. J. Kertes and T. M. Johnson, Evidence-based eye care. Philadelphia: Lippincott Williams \& Wilkins, 2007.

[2] I. Goodfellow, Y. Bengio, and A. Courville, Deep Learning, "Regularization for deep learning", MIT Press, 2016, ch. 7, sec 7.8, pp. 241-249.

[3] Ronneberger O., Fischer P., Brox T. (2015) U-Net: Convolutional Networks for Biomedical Image Segmentation. In: Navab N., Hornegger J., Wells W., Frangi A. (eds) Medical Image Computing and Computer-Assisted Intervention - MICCAI 2015. MICCAI 2015. Lecture Notes in Computer Science, vol 9351. Springer, Cham 


\section{Questions}

(C) A. Lochbihler 2019

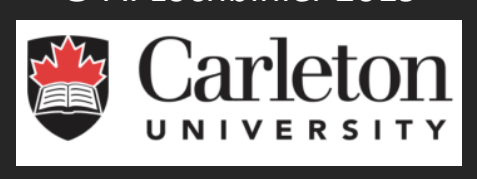

